\title{
Comparaison between the method which is used the spatial contextual information and some methods of image classifi- cation
}

Houda Hassouna

LARGHYDE Laboratory, University of Biskra, Biskra, Algeria.

\begin{abstract}
In this paper, we present the results obtained for the remote sensing image classification by using three methods of classification namely, Gaussian process classification method (GPC), morphological profile for classification method (MPC) and spatial contextual Gaussian process classification method (SGPC). Several classification approaches have shown that the exploitation of spatial contextual information can be attractive to increase the classification accuracy by introducing a new automated learning approach based on Gaussian process theory.
\end{abstract}

Keywords: Gaussian process, morphological profile, spatial contextual Gaussian process classification, spatial contextual information.

2010 MSC: 60G15, 62M30.

(C)2019 All rights reserved.

\section{Introduction and preliminaries}

To the best of our knowledge, the integration of spatial contextual information in a GPC model has not yet been envisioned for classifying remote sensing imagery. An apparently close work can be found in Jun and Ghosh [5], where GP regression is used to exploit spatial coordinates of the training samples for predicting mean vectors. The classification task is performed by means of a maximum likelihood (ML) classifier. In our case, we exploit spatial contextual information, which is different from spatial coordinates, and embed it in a GPC model. It is well-known that spatial contextual information can be useful, if well exploited, to improve the classification accuracy by opportunely capturing local spatial correlation conveyed in the image under analysis. This letter aims thus at introducing a new mathematical formulation of GPC that integrates spatial contextual information in the Bayesian classification model.

Assuming a spatial neighborhood system of size $\mathrm{N}^{*} \times \mathrm{N}^{*}$, let us consider a training set $\mathbf{\mathrm { D }}=(\grave{\mathbf{X}}, \grave{\mathbf{y}})$ consisting of a matrix of training data $\grave{\mathbf{X}}=\left[\begin{array}{l}\mathbf{X} \mathbf{X}_{\mathfrak{n}}^{*}\end{array}\right]$ accompanied with labels $\grave{\mathbf{y}}=\left[\mathbf{y} \mathbf{y}_{\mathfrak{n}}^{*}\right]$, where $\mathbf{y}_{\mathfrak{n}}^{*}$ and $\mathbf{X}_{\mathrm{n}}^{*}$ are the spatial neighbors of $\mathbf{y}_{*}$ and $\mathbf{x}_{*}$. We aim at determining the label $\mathbf{y}_{*}$ at new test point $\mathbf{x}_{*}$ by computing the class posterior probability $\mathrm{P}\left(\mathrm{y}_{*} \mid \grave{\mathbf{D}}, \mathbf{x}_{*}\right)$,

$$
\mathrm{P}\left(\mathrm{y}_{*}=+1 \mid \grave{\mathbf{D}}, \mathbf{x}_{*}\right)=\int \mathrm{P}\left(\mathrm{y}_{*} \mid \mathrm{f}_{*}\right) \mathrm{P}\left(\mathrm{f}_{*} \mid \grave{\mathbf{D}}, \mathbf{x}_{*}\right) \mathrm{d} \mathrm{f}_{*} .
$$

Email address: houda.hassouna@yahoo.fr (Houda Hassouna)

doi: $10.22436 / \mathrm{mns} .05 .01 .02$

Received: 2017-11-20 Revised: 2018-12-19 Accepted: 2019-05-04 
The prediction of the point $\mathbf{x}_{*}$ is evaluated by exploiting the Gaussian approximation in Eq. (1.1)

$$
\mathrm{P}\left(\mathrm{y}_{*}=+1 \mid \grave{\mathbf{D}}, \mathbf{x}_{*}\right) \simeq \mathrm{q}\left(\mathrm{y}_{*}=+1 \mid \grave{\mathbf{D}}, \mathbf{x}_{*}\right)=\int \mathrm{P}\left(\mathrm{y}_{*} \mid \mathbf{f}_{*}\right) \mathrm{q}\left(\mathrm{f}_{*} \mid \grave{\mathbf{D}}, \mathbf{x}_{*}\right) \mathrm{df}_{*},
$$

where $\mathrm{q}\left(\mathbf{f}_{*} \mid \grave{\mathbf{D}}, \mathbf{x}_{*}\right)$ is Gaussian with mean and variance given as

$$
\left\{\begin{array}{l}
\mu_{*}=\grave{k}_{*}^{\top} \grave{K}^{-1} \hat{\mathbf{f}}^{\prime} \\
\sigma_{*}^{2}=\mathrm{k}\left(\mathbf{x}_{*}, \mathbf{x}_{*}\right)-\grave{k}_{*}^{\top}\left(\grave{K}+\grave{W}^{-1}\right)^{-1} \grave{k}_{* \prime}
\end{array}\right.
$$

where $\grave{K}$ is the covariance matrix and $\grave{k}_{*}^{\top}=\left[k\left(\mathbf{x}_{1}, \mathbf{x}_{*}\right) k\left(\mathbf{x}_{2}, \mathbf{x}_{*}\right) \cdots \mathrm{k}\left(\mathbf{x}_{\mathrm{N}}, \mathbf{x}_{*}\right) \mathrm{k}\left(\mathbf{x}_{\mathrm{N}+1}, \mathbf{x}_{*}\right) \cdots \mathrm{k}\left(\mathbf{x}_{\grave{\mathrm{N}}}, \mathbf{x}_{*}\right)\right]$ is a vector of kernel distances (covariances) between $\mathbf{x}_{*}$ and all the training and neighborhood samples.

\section{Main results}

\subsection{Data set}

We used an image acquired over an urban area, this image was acquired over a part Boumerdes city (Algeria) in 2002 by the Quickbird sensor with a resolution of $1 \mathrm{~m}$. It is characterized by four channels (Red, Green, Blue, and Near infrared). The ground truth includes nine thematic classes, namely, water, sand, trees, asphalt, pavement, rocks, roof1 (tile roof), roof2 (cement roof), and bare soil. Table 1 lists the numbers of training and test samples used for each class.

Table 1: Numbers of training and test samples used in experiments.

\begin{tabular}{|l|c|c|}
\hline Class name & Numbers of training samples & Numbers of test samples \\
\hline 1- Water & 600 & 2400 \\
\hline 2- Sand & 600 & 2400 \\
\hline 3- Trees & 375 & 700 \\
\hline 4- Pavement & 105 & 200 \\
\hline 5- Asphalt & 343 & 500 \\
\hline 6- Rocks & 175 & 450 \\
\hline 7- Roof1 & 75 & 200 \\
\hline 8- Roof2 & 294 & 500 \\
\hline 9- Bare soil & 300 & 700 \\
\hline Total & $\mathbf{2 8 6 7}$ & $\mathbf{8 0 5 0}$ \\
\hline
\end{tabular}

In all experiments, the covariance function adopted is the well-known squared exponential covariance function. The hyperparameters of the models for both the standard GPC and the proposed SGPC classifiers were estimated according to the procedure based on the maximization of the log marginal likelihood as described in Rasmussen and Williams [9].

Classification performance was evaluated in terms of three measures, which are:

1) overall accuracy (OA), which is the percentage of correctly classified pixels among all the pixels considered (independently of the classes they belong to);

2) average accuracy (AA), which is the average over the classification accuracies obtained for the different classes;

3) class-specific accuracy, which is the percentage of correctly classified pixels among the pixels of the considered class.

\subsection{The comparison between SGPC method and Standard GPC method}

At first, we performed experiments by considering a neighborhood system of 3 pixels $\times 3$ pixels. In particular, we run the proposed SGPC method up to convergence. As it can be seen in Figures 1, 2, and 3, convergence was achieved at third iterations. The main improvement was obtained at the first iteration, and then the accuracy stabilized. 


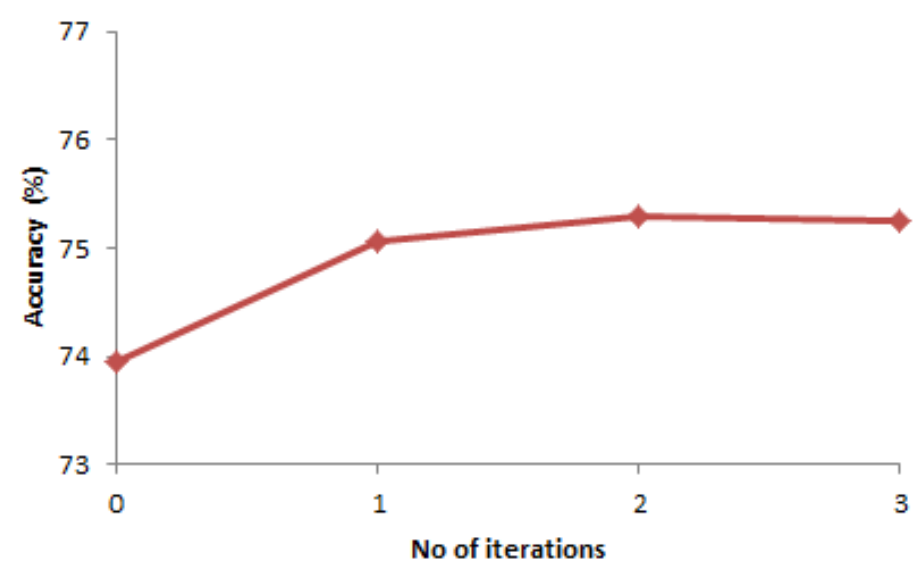

Figure 1: Overall accuracy achieved on the test samples by the investigated SGPC classifier versus the number of iterations (3 pixels $\times 3$ pixels).

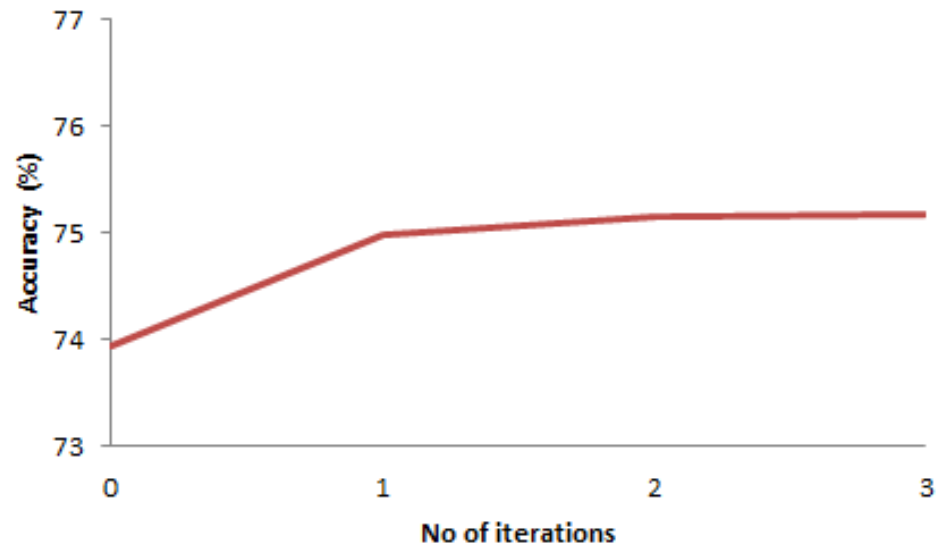

Figure 2: Overall accuracy achieved on the test samples by the investigated SGPC classifier versus the number of iterations ( 5 pixels $\times 5$ pixels).

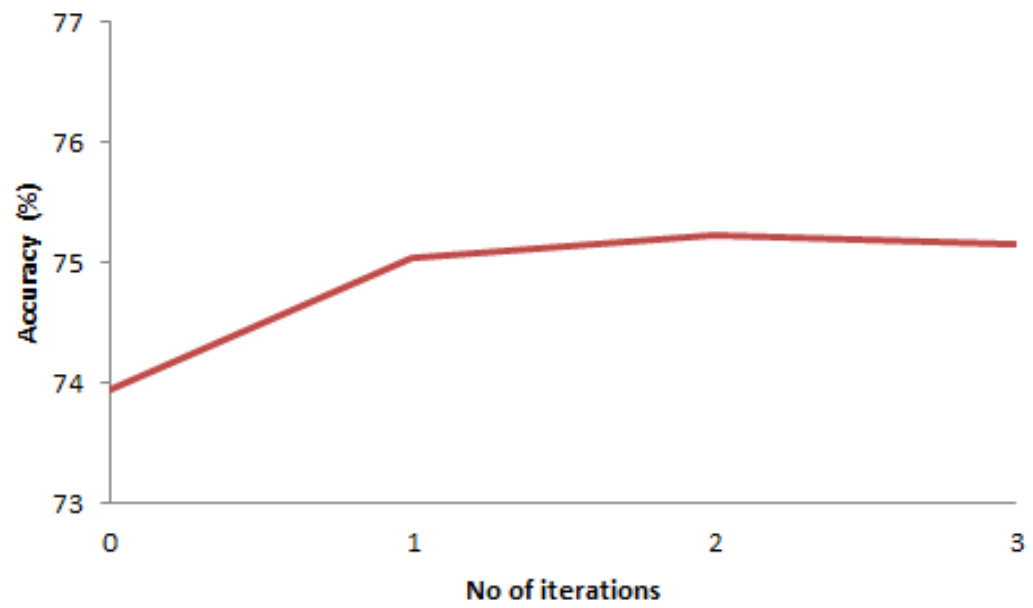

Figure 3: Overall accuracy achieved on the test samples by the investigated SGPC classifier versus the number of iterations (7 pixels $\times 7$ pixels). 
The detailed results achieved at convergence are reported in Tables 2, 3, and 4. Compared to the standard SGPC, we have

1) an improvement of about $1.12 \%$ in $\mathrm{OA}$ and $1.35 \%$ in AA when neighborhood system of 3 pixels $\times 3$ pixels;

2) an improvement of about $1.34 \%$ in $\mathrm{OA}$ and $1.61 \%$ in AA when neighborhood system of 5 pixels $\times 5$ pixels;

3) an improvement of about $1.20 \%$ in $\mathrm{OA}$ and $1.50 \%$ in $\mathrm{AA}$ when neighborhood system of 7 pixels $\times 7$ pixels.

Most of the classes take profit from the exploitation of spatial contextual information, and in particular

1) the class 'roof2' for which a boost of more than $4 \%$ is observed when neighborhood system of 3 pixels $\times 3$ pixels;

2) the class 'roof2' for which a boost of more than $5 \%$ is observed when neighborhood system of 5 pixels $\times 5$ pixels;

3) the class 'roof2' for which a boost of more than $5 \%$ is observed when neighborhood system of 7 pixels $\times 7$ pixels.

Table 2: Accuracies achieved by the investigated classifiers on the test samples (iteration 1).

\begin{tabular}{|c|c|c|c|c|c|}
\hline & \multirow[t]{2}{*}{ GPC method } & \multicolumn{3}{|c|}{ SGPC method (iteration 1) } \\
\hline & & & $3 \times 3$ size & $5 \times 5$ size & $7 \times 7$ size \\
\hline \multicolumn{2}{|l|}{ OA (\%) } & 73.95 & 75.07 & 74.98 & 75.03 \\
\hline \multicolumn{2}{|l|}{$\mathrm{AA}(\%)$} & 66.42 & 67.77 & 67.58 & 67.82 \\
\hline \multirow{9}{*}{$\begin{array}{c}\text { Class-specific } \\
\text { accuracies (\%) }\end{array}$} & 1 & 100 & 99.00 & 98.96 & 99.00 \\
\hline & 2 & 61.42 & 63.58 & 63.54 & 63.25 \\
\hline & 3 & 93.57 & 95.71 & 95.71 & 95.43 \\
\hline & 4 & 42.50 & 46.00 & 45.50 & 46.50 \\
\hline & 5 & 85.40 & 85.40 & 85.40 & 85.40 \\
\hline & 6 & 34.75 & 36.89 & 36.22 & 36.67 \\
\hline & 7 & 70.50 & 71.00 & 70.50 & 70.50 \\
\hline & 8 & 67.20 & 71.40 & 71.40 & 71.80 \\
\hline & 9 & 42.43 & 41.00 & 41.00 & 41.86 \\
\hline
\end{tabular}

Table 3: Accuracies achieved by the investigated classifiers on the test samples (iteration 2).

\begin{tabular}{|c|c|c|c|c|c|}
\cline { 3 - 6 } \multicolumn{2}{c|}{} & GPC method & \multicolumn{3}{|c|}{ SGPC method (iteration 2) } \\
\cline { 3 - 6 } \multicolumn{2}{c|}{} & & $3 \times 3$ size & $5 \times 5$ size & $7 \times 7$ size \\
\hline \multicolumn{2}{|c|}{ OA (\%) } & 73.95 & 75.29 & 75.15 & 75.22 \\
\hline \multirow{2}{*}{ AA (\%) } & 66.42 & 68.03 & 67.96 & 68.14 \\
\hline \multirow{4}{*}{$\begin{array}{c}\text { Class-specific } \\
\text { accuracies (\%) }\end{array}$} & 1 & 100 & 99.04 & 99.08 & 99.13 \\
\cline { 2 - 6 } & 2 & 61.42 & 63.83 & 63.46 & 63.25 \\
\cline { 2 - 6 } & 3 & 93.57 & 95.71 & 95.86 & 96.14 \\
\cline { 2 - 6 } & 5 & 42.50 & 45.50 & 46.00 & 46.00 \\
\cline { 2 - 6 } & 6 & 34.40 & 85.60 & 85.80 & 85.80 \\
\cline { 2 - 6 } & 7 & 70.50 & 36.22 & 35.78 & 36.22 \\
\cline { 2 - 6 } & 8 & 67.20 & 72.00 & 72.00 & 72.50 \\
\cline { 2 - 6 } & 9 & 42.43 & 42.00 & 41.80 & 72.60 \\
\hline
\end{tabular}


Table 4: Accuracies achieved by the investigated classifiers on the test samples (iteration 3).

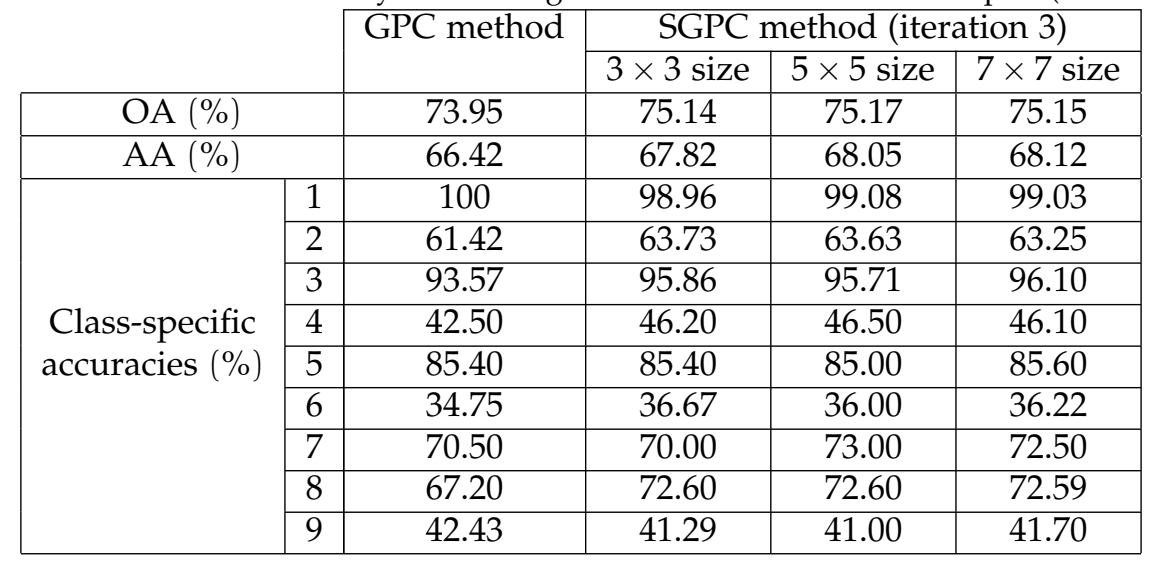

Finally, in order to analyze the impact of the size of the neighborhood system on the classification results, we repeated the previous experiments by adopting increasing values for the window size, namely 5 pixels $\times 5$ pixels and 7 pixels $\times 7$ pixels. The convergence was achieved in all cases at the third iteration. The overall accuracies yielded by the SGPC method are plotted in Figures 4, 5, and 6, which suggests that the size of the neighborhood is not critical.

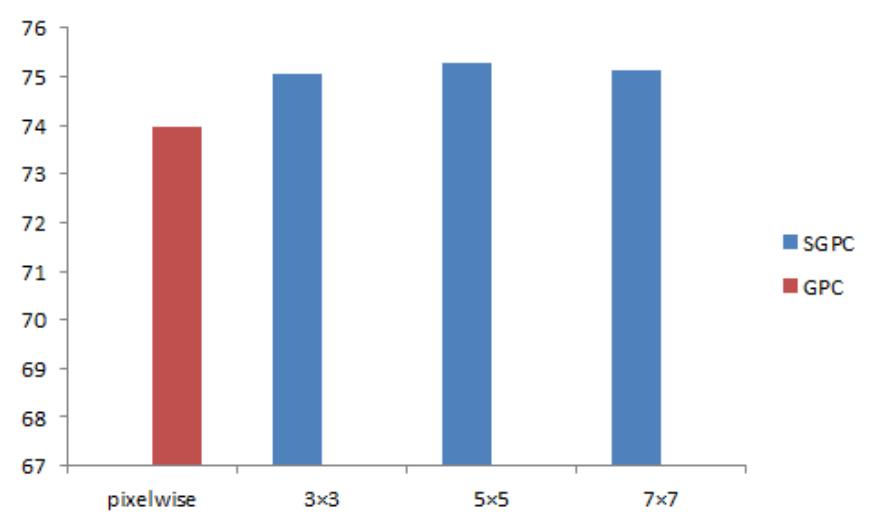

Figure 4: Overall accuracy achieved on the test samples by the investigated SGPC classifier versus the size of the neighborhood system."Pixelwise" stands for the standard GPC classifier (iteration 1).

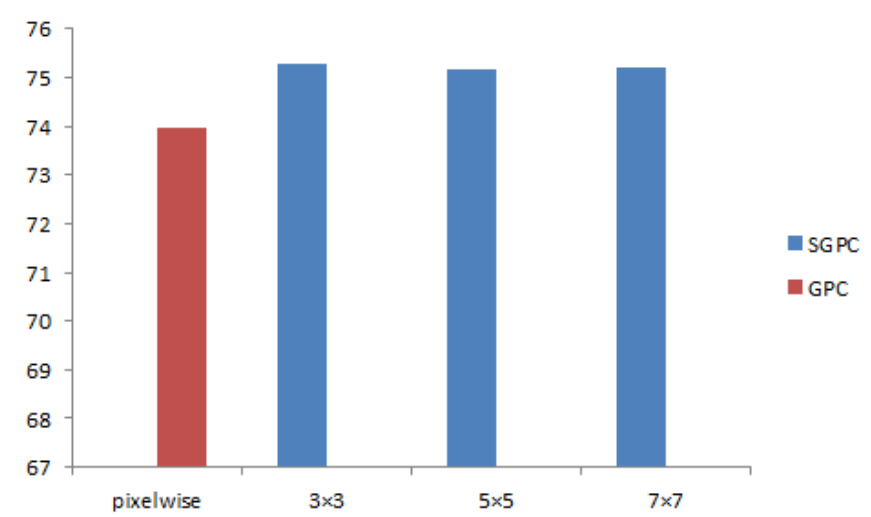

Figure 5: Overall accuracy achieved on the test samples by the investigated SGPC classifier versus the size of the neighborhood system. "Pixelwise" stands for the standard GPC classifier (iteration 2). 


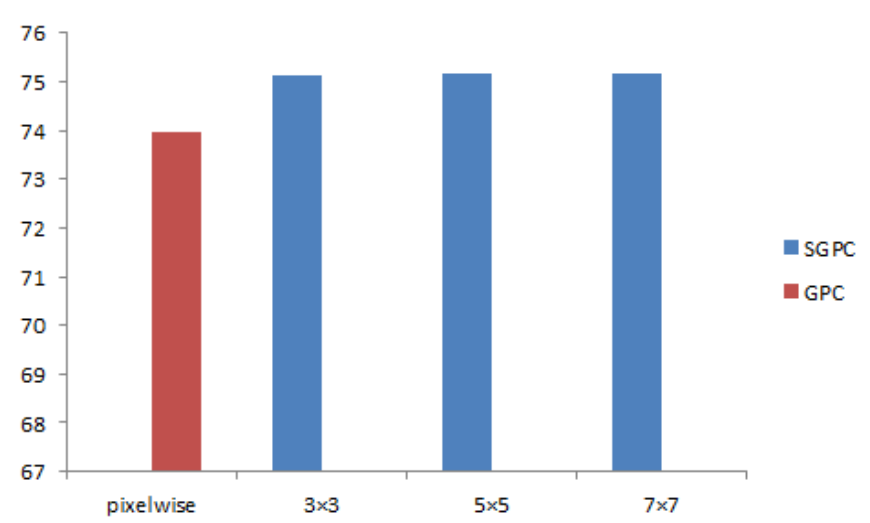

Figure 6: Overall accuracy achieved on the test samples by the investigated SGPC classifier versus the size of the neighborhood system. "Pixelwise" stands for the standard GPC classifier (iteration 3).

\subsection{The comparison between SGPC method and MP-GPC method}

For the sake of comparison, we run the GPC classifier fed with an additional set of 8 morphological profile (MP) features, concatenated with the 4 original features (in total 12 features).

The MP was generated by applying opening and closing operations with a square-shape structuring element. The overall accuracies yielded by the SGPC and the MP-GPC methods are plotted in Fig. 7, which suggests that: 1) the size of the neighborhood is not critical for SGPC, and 2) SGPC outperforms MP-GPC Table 5.

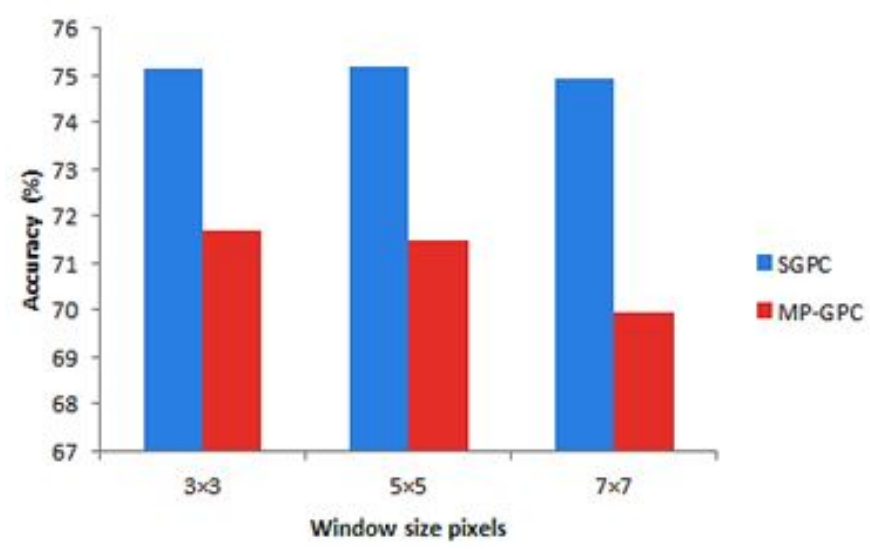

Figure 7: Overall accuracy achieved on the test samples by the investigated SGPC classifier versus the standard MP-GPC classifier.

Table 5: Accuracies achieved by the investigated classifiers (SGPC, MP-GPC) on the test samples.

\begin{tabular}{|c|c|c|c|}
\cline { 3 - 4 } \multicolumn{2}{|c|}{} & MP method & SGPC method \\
\hline \multicolumn{2}{|c|}{ OA (\%) } & 71.69 & 75.95 \\
\hline \multicolumn{2}{|c|}{ AA (\%) } & 65.00 & 67.61 \\
\cline { 2 - 4 } & 1 & 98.63 & 98.96 \\
\cline { 2 - 4 } & 2 & 57.08 & 63.63 \\
\cline { 2 - 4 } Class-specific & 3 & 78.86 & 95.86 \\
\cline { 2 - 4 } accuracies (\%) & 4 & 37.50 & 46.00 \\
\cline { 2 - 4 } & 5 & 90.20 & 85.40 \\
\cline { 2 - 4 } & 6 & 31.33 & 36.67 \\
\cline { 2 - 4 } & 7 & 77.50 & 70.00 \\
\cline { 2 - 4 } & 8 & 68.80 & 72.60 \\
\cline { 2 - 4 } & 9 & 45.14 & 41.29 \\
\hline
\end{tabular}




\section{Conclusion}

We will look at the improvement of the GPC capabilities so as to integrate spatial contextual information, in order to improve the accuracy of the classification. Experimental results show that the SGPC can help improve the classification accuracy compared to Both GPC and MPC. Finally, this paper, through careful examination of the spatial contextual classification method, sheds light on the effectiveness of spatial contextual information on image classification.

\section{References}

[1] Y. Bazi, F. Melgani, Classification of Hyperspectral Remote Sensing Images Using Gaussian Processes, IEEE Trans. Geosci. Remote Sensing Symposium, 2008 (2008), 2 pages.

[2] Y. Bazi, F. Melgani, Gaussian process approach to remote sensing image classification, IEEE Transactions on Geoscience and Remote Sensing, 48 (2009), 186-197.

[3] H. Hassouna, F. Melgani, Comparison between spatial contextual Gaussian process classification method and other methods, The conference ADAM (Errachidia, Morroco), 2017 (2017), 12 pages.

[4] H. Hassouna, F. Melgani, Z. Mokhtari, Spatial Contextual Gaussian Process Learning for Remote Sensing Image Classification, Remote Sensing Lett., 6 (2015), 519-528.

[5] G. Jun, J. Ghosh, Spatially Adaptive Classication of Hyperspectral Data with Gaussian Processes, in IEEE International Geoscience and Remote Sensing Symposium, 2 (2009). 1

[6] C. E. Rasmussen, Evaluation of Gaussian Processes and Other Methods for Non-Linear Regression, Ph.D. thesis (University of Toronto), ProQuest LLC, Ann Arbor, (1997).

[7] C. E. Rasmussen, Z. Ghahramani, Infinite Mixtures of Gaussian Process Experts, Proceeding NIPS'01 Proceedings of the 14th International Conference on Neural Information Processing Systems: Natural and Synthetic, 2001 (2001), 881-888.

[8] C. E. Rasmussen, M. Kuss, Gaussian Processes in Reinforcement Learning, Proceeding NIPS'03 Proceedings of the 16th International Conference on Neural Information Processing Systems, 2003 (2003), 751-758.

[9] C. E. Rusmassen, C. K. I. Williams, Gaussian Process for Machine Learning, MIT Press, Cambridge, (2005). 2.1 